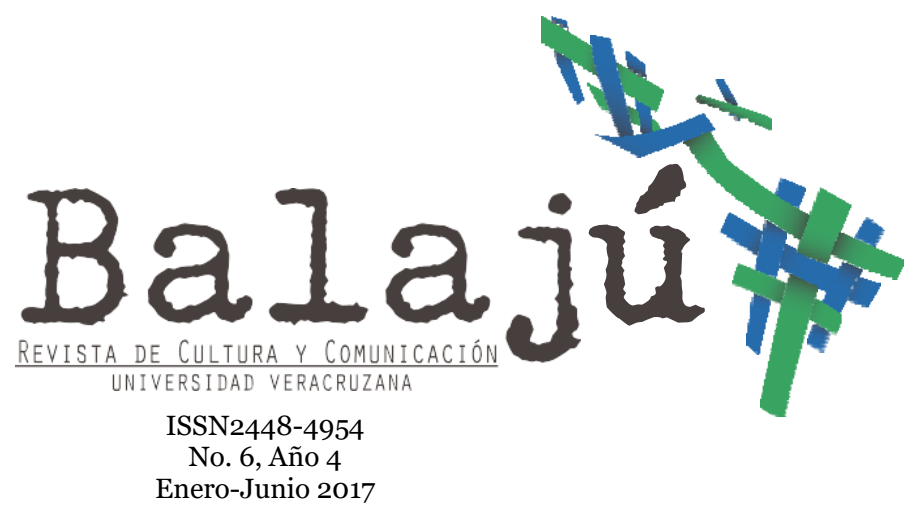

\title{
Identidades indígenas en Quintana Roo. Una mirada a sus configuraciones en
} universidades con enfoque intercultural

Indigenous Identities in Quintana Roo: Configurations in Intercultural University Contexts

Luis Ernesto Solano Becerril

Universidad Autónoma de Aguascalientes

luisolano23@yahoo.com.mx

\section{RESUMEN}

En este artículo se intenta mostrar los diferentes elementos que utilizan los estudiantes para configurar su identidad indígena o mestiza. La investigación se lleva a cabo en instituciones de educación superior con enfoque intercultural en el estado de Quintana Roo como parte del trabajo de tesis doctoral. Primeramente, se discutirá la metodología oficial que identifica la identidad indígena bajo características específicas y generales para, posteriormente, dar a conocer las formas en que se expresa en la vida cotidiana como resultado de un proceso más complejo y hasta estratégico por parte de los estudiantes.

\begin{abstract}
This article explores the different components that students draw upon to configure their indigenous or mestizo identities. Based on doctoral research carried out in universities with an intercultural orientation in the state of Quintana Roo, it begins with a discussion of official methodologies that classify indigenous identity according to certain external characteristics; it then goes on to show the different ways in which indigenous identity is expressed in students' everyday life as a result of more complex and even strategic processes.
\end{abstract}

\section{PALABRAS CLAVES}

Identidad, interculturalidad, procesos identitarios, identidad intercultural.

\section{KEYWORDS}

Identity, interculturality, identity processes, intercultural identity. 


\title{
Identidades indígenas en Quintana Roo. Una mirada a sus configuraciones en universidades con enfoque intercultural
}

\author{
Luis Ernesto Solano Becerril ${ }^{1}$
}

\section{Introducción}

En México la educación intercultural se crea oficialmente en 2001 con la fundación de la Coordinación General de Educación Intercultural Bilingüe (CGEIB) que depende directamente de la Secretaría de Educación Pública (Olivera Rodríguez, 2014). A partir de este decreto se empiezan a generar escuelas con enfoque intercultural y, a pesar de que los estudiantes indígenas asisten a todas las modalidades y niveles, la educación intercultural bilingüe se ofrece sólo en las escuelas indígenas, en los niveles preescolar y primaria (Mendoza, 2017). La educación superior, aunque no es obligatoria, no es la excepción en implementar el enfoque, cuyo origen no parte de la autonomía de los pueblos originarios sino de la relación dialógica y armónica entre indígenas y no indígenas (Mateos y Dietz, 2016) y supone la interculturalización del currículo oficial a través de aprendizajes significativos situados en el reconocimiento de prácticas, conocimientos y saberes de los pueblos indígenas para, a partir de éstos, mirar lo universal (López, citado en Mendoza, 2017: 55).

La educación intercultural se impulsa como la forma más acorde con un mundo que está en diálogo entre culturas distintas. Con ella se podría disminuir la desigualdad e influir en la trasformación de las relaciones interétnicas mediante el desarrollo de una educación más justa y menos discriminatoria, que pueda atender las demandas educativas de la población indígena que no haya tenido acceso a la educación y, al mismo tiempo, proteger a las culturas indígenas de una eventual pérdida de identidad (Rebolledo, 2005). Pero cabe preguntarse, ¿qué es la identidad indígena que se trata de proteger, preservar y fomentar? ¿Cómo son expresadas por los estudiantes de estas instituciones? Para ello necesitamos saber cómo se define la identidad, debido a que su fortalecimiento, promoción y desarrollo son objetivos a cumplir en esta modalidad educativa implementada por el Centro de Estudios Interculturales (Cenei) de la Universidad de Quinata Roo (UQRoo) y la Universidad Intercultural Maya de Quintana Roo (UIMQRoo). Sin comprender cómo se forma la identidad indígena, el sistema intercultural podría presentar inconsistencias, por lo que éste debe de ser su punto de partida y es el objetivo de este artículo.

\section{Identidad étnica, ¿se puede medir?}

Este término es sumamente importante pues, al hablar de interculturalidad, se está hablando de una relación entre varias culturas e identidades, por lo que es necesario definirlo teóricamente para luego analizarlo en un contexto real. La identidad étnica es un concepto clave para entender significados, interacciones, culturas, cosmovisiones, etc., y también indica el sentimiento de pertenencia o afinidad a un grupo humano. Este término es capaz de reconocer el lugar que el grupo juega en la

${ }^{1}$ Universidad Autónoma de Aguascalientes luisolano23@yahoo.com.mx 
historia, la función e importancia del lenguaje, la construcción de subjetividad, los discursos posicionados en un espacio y tiempo particulares con base en la diferencia (Hall, 2014: 342). Es necesario comprender que la identidad étnica se constituye a partir de un proceso de contrastación, pero sobre todo de confrontación con el otro, razón por la cual no se puede analizar independientemente de las relaciones intra e interétnicas, porque esos son los espacios de interacción temporal donde se mantiene, se actualiza y se renueva (Bari, 2002: 156).

Con estas ideas como base, se han desarrollado diferentes métodos que nos puedan dar indicadores para definir la identidad étnica. En Europa se ha desarrollado el ethnosizer, cuyo objetivo es medir la intensidad de la pertenencia y conocer qué tan asimilados, integrados, marginados o separados están los individuos y grupos respecto de la cultura mayoritaria a través de datos como el lenguaje, la cultura, las interacciones sociales, la historia de migración y la autoidentificación. Este instrumento ha servido como sustento para perfeccionar, desarrollar y aplicar más modelos de medición en ese contexto (Epstein y Heizler, 2015). En ejercicios previos también se intentó mediante un cuestionario en que los componentes eran más complejos: lenguaje, amistad, funcionalidad del grupo, tradiciones, autorreconocimiento, afectividad y moralidad, entre otros (Espín et al., 1998). Cabe recordar que las relaciones interétnicas en Europa están orientadas a integrar o asimilar a las poblaciones inmigrantes de otras regiones.

En América Latina el enfoque utilizado está orientado a las relaciones con poblaciones originarias y esto representa un cambio importante en la forma de identificar los distintos grupos étnicos, ya que de por medio está la generación de políticas públicas y la designación de recursos económicos. La discusión parece ser más amplia y se han generado indicadores estructurales, de procesos y de resultados que, a la vez, se subdividen en elementos territoriales, patrimonio cultural colectivo, organización social, identidad colectiva y de género, modelos de autonomía o autogobierno, entre otros más (UNAM, 2008). Así, cada país tiene su propia forma de identificar las identidades étnicas; Bolivia es el país en el que habita mayor población originaria (62.2\%), seguido por Guatemala (41\%), Perú (24\%) y México (15\%), mientras que el agregado en Centro y Suramérica es de 826 pueblos indígenas (Cepal, 2014), pero para el tema que se trata pondremos especial atención en México.

\section{Metodología oficial mexicana para definir la identidad étnica}

Según el Instituto Nacional de Estadística y Geografía (INEGI), la identidad étnica de una persona tiene un carácter dinámico que se modifica y se redefine en el curso de la vida de un individuo, ya sea por migración, estrato social, cambio de entorno o el periodo histórico (INEGI, s/f). En el último censo de población realizado en 2010, la población perteneciente a pueblos originarios se identificó con base en dos criterios: $a$ ) que la persona hablara alguna lengua indígena a partir de los 3 años y b) que la persona se autoadscribiera debido a sus propias concepciones. Dicha metodología se conservó casi íntegra para la encuesta intercensal hecha cinco años después, pero hay un cambio drástico en los resultados, pues mientras que en 2010 se contabilizaba como tal 14.9\% de la población, en 2015 disminuyó a $6.5 \%$ (INEGI, 2010a; 2015), reflejando una caída brusca de 8.4\%, pero sin indicar las posibles causas. 
Los criterios de lengua y autoadscripción no parecen ser la mejor opción para definir el origen étnico, ya que el supuesto de que alguien que hable lengua indígena sea por definición un indígena es falseable, pues existen casos de personas que hablan alguna lengua y no pertenecen a una etnia y a la inversa; como ejemplo, están las 400000 personas que son hablantes de lengua indígena y no se consideran indígenas (Gutiérrez y Valdés, 2015: 58). Un ejemplo de ello es la Encuesta Nacional de Indígenas, en la que se pregunta sobre el tipo de indicadores, los que enmarcan esta identidad étnica, y cuyos resultados obtenidos por porcentaje aprobatorio son:

Gráfica 1. Indicadores que pudieran enmarcar el ser indígena

Nació en comunidad indígena y habla la lengua

Ambos padres son indígenas y no habla la lengua

Nació en una comunidad y no habla la lengua

Habla la lengua

Sigue las costumbres y no habla la lengua

Cuando el padre lo es y la madre no

Cuando la madre es indígena y el padre no

O
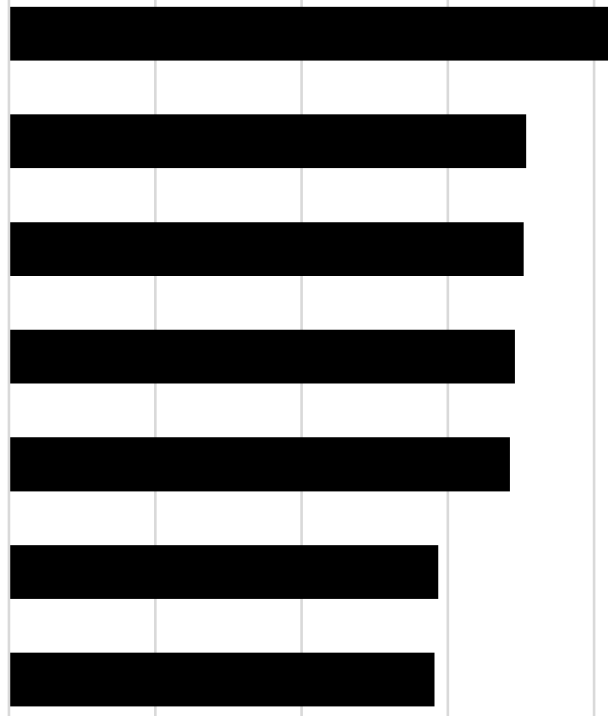

20

40

60

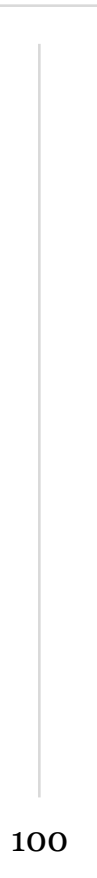

FUENTE: Gutiérrez y Valdés, 2015: 59.

El espacio geográfico es una variable que casi no se toma en cuenta pero que tiene influencia. Si bien la mayoría de pueblos originarios radica en contextos rurales, la identidad étnica indígena en la ciudad prácticamente está escondida, negada o se usa a conveniencia (Bravo, 1993) y tiende a debilitarse porque no hay lugares para su ejercicio, lo que no permitiría entonces observar o tomarlos en cuenta en las estadísticas oficiales. Es muy posible suponer la coexistencia de indígenas con quienes no lo son, incluso los padres pudieran identificarse sin que los hijos reproduzcan sus pautas culturales ni se asuman como tales, por lo que es necesario empezar a tomar en cuenta más aspectos para su definición, como la relación de parentesco, la conformación de las familias, el tipo de localidad de residencia, entre otros (Hernández y Chávez, 2007), como se pudo observar en la tabla anterior.

Aunado a esta complejidad, la identidad no sólo puede ser numérica o la suma de diversos indicadores: también contiene una dimensión cualitativa que se forma, se mantiene y se manifiesta en y por los procesos de interacción y comunicación social (Habermas, citado en Giménez, 2007: 23), información que es difícil recuperar en cuestionarios y estadísticas oficiales. En comparación con la identidad indígena, 
no hay indicadores claros que puedan definir la identidad mestiza; por lo general, no se le considera como una etnia debido a que tiene un proceso más avanzado de homogeneización, aunque guarda sus particularidades y diferencias de cultura conocidas como culturas regionales (sur, centro y norte) y es el grupo étnico mayoritario, dominante en todos los órdenes y a tal grado que se le identifica como nación misma, por lo cual no se cuestiona su pertenencia étnica pues, al ser mayoría, se le considera como lo normal (Durán, 2005: 97).

Podemos ver que la identidad étnica es difícil de definir por las distintas variables que están interrelacionadas y porque está en función del tipo de relaciones sociales que establecen las personas y los contextos en que se desenvuelven y en los que tratan de integrarse de alguna forma (Bravo, 1993). El análisis del tema es relevante, ya que la identidad indígena es un tópico que está presente en temas de interculturalidad en México y, específicamente, en el ámbito educativo que utiliza esta modalidad, pues el contacto e intercambio entre culturas distintas es algo que se desea.

\section{Elementos que conforman la identidad en estudiantes de universidades de Quintana Roo}

En la investigación empírica se deseó conocer la forma en que las culturas entran en contacto por medio de las identidades y cómo se enriquecen unas a otras. Para ello se diseñó una metodología cualitativa, pues nos interesa recuperar las subjetividades, voces y experiencias de quienes viven la interculturalidad, lo cual los cuestionarios no alcanzan a visualizar. Se utilizaron como técnicas principales la entrevista estructurada y los grupos focales aplicados a estudiantes.

En la UQRoo existen estudiantes con identidad indígena en todas las licenciaturas impartidas, pero el enfoque intercultural no está aplicado a toda la institución, únicamente en el Cenei, que para 2015 tenía 838 alumnos de origen indígena, de los cuales 825 eran mayas y los 13 restantes fueron identificados como mestizos, mixteco, totonaco, zapoteco, kanjobal, chol y tsotsil (Cenei, 2016).

En el desarrollo teórico era relativamente clara la forma de identificar a los estudiantes que pertenecían a culturas originarias, específicamente la maya, basándose en indicadores oficiales que catalogan bajo características de lengua, autoadscripción o, en ocasiones, ciertos usos y costumbre; sin embargo, esta tarea en particular fue más complicada en el ámbito urbano de Quintana Roo, ya que no hubo objetos culturales expresados a simple vista, como la ropa, ni tampoco el uso de la lengua materna en el espacio público diario.

Se pudo visualizar que la identidad indígena es ocultada y negada en la universidad y en la ciudad (o es ejercida en espacios muy específicos), debido a la historia de deslegitimación de la cultura maya:

Dentro del contexto de Quintana Roo esto se hace visible en la interacción cotidiana, en donde hablar maya es un elemento para mostrar la identidad pero, al mismo tiempo, para ser considerado un "mayita", que es un término específico para referirse a toda la población de origen indígena de forma despectiva y se les considera ignorantes y tontos, y automáticamente son catalogados como inferiores. Por esto, los jóvenes dejan de hablar su lengua, como una forma de autodefensa y así evitar evidenciarse, empiezan a querer transitar de ser una persona de localidad rural a una de ciudad, negando sus orígenes étnicos. En este proceso las escuelas tienen su papel aunque sea de forma no intencional, pues a partir de 


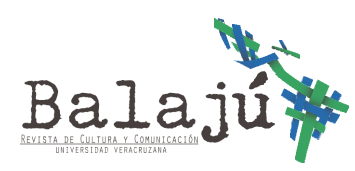

secundaria los jóvenes tienen que salir a las ciudades a estudiar, y es en estos espacios públicos donde se empieza a abandonar lo maya (Chi Canul, 2010).

Esta práctica de negación ya ha sido visibilizada con anterioridad y pareciera que es la más común en la ciudad, debido a que lo indígena no se identifica con la modernidad, y se vuelve objeto de diversas agresiones de tipo cultural e ideológico, y como estrategia de defensa se deja de ser indígena para dejar de ser agredido (Bravo, 1993). Todo este conjunto de acciones genera una dinámica de exclusión social en general y tiene sus reflejos dentro del sistema educativo, en donde los estudiantes también ocultan su cultura y su identidad originaria.

Con estas dificultades, se diseñó una muestra no representativa -pues nunca se tuvo certeza de las identidades expresadas en el espacio público- y que estuvo formada por dos grupos de ocho estudiantes voluntarios. Un primer conjunto que participa continuamente en todas las actividades y que fue recomendado por los académicos del Cenei y un segundo que está inscrito en asesorías de lengua maya del mismo centro. Con base en los datos obtenidos, se pudo construir una tipología de la identidad indígena que es conformada por la autoadscripción, el arraigo a la localidad, ancestralidad, el lugar de origen y la lengua. Uno de los descubrimientos más importantes es el hecho de que no necesariamente al ser indígena se tiene una afinidad con la cultura originaria, lo que genera una ruptura entre una y otra categoría, lo que se ilustra a continuación:

Tabla 1. Elementos que configuran la identidad en estudiantes de la Universidad de Quintana Roo

\begin{tabular}{|c|c|c|c|c|c|c|}
\hline \multicolumn{5}{|c|}{ Elementos que conforman la identidad de los estudiantes } & Identidad & $\begin{array}{l}\text { Afinidad } \\
\text { cultural }\end{array}$ \\
\hline Autoadscripción & $\begin{array}{l}\text { Arraigo a } \\
\text { la } \\
\text { localidad² }\end{array}$ & Ancestralidad 3 & $\begin{array}{l}\text { Lugar de } \\
\text { origen } 4\end{array}$ & $\begin{array}{l}\text { Lengua } \\
\text { indígena }\end{array}$ & & \\
\hline Indígena & $\checkmark$ & $\checkmark$ & Rural & $\checkmark$ & Indígena & \multirow{4}{*}{ Indígena } \\
\hline Indígena & $\sqrt{ }$ & $\sqrt{ }$ & Ciudad & $\mathrm{X}$ & Indígena & \\
\hline Mestizo & $\checkmark$ & $\checkmark$ & Rural & $\mathrm{X}$ & Mestiza & \\
\hline Mestizo & $\sqrt{ }$ & $\mathrm{X}$ & Ciudad & $\sqrt{ }$ & Mestiza & \\
\hline Indígena & $\mathrm{X}$ & $\checkmark$ & Rural & $\mathrm{X}$ & Indígena & \\
\hline Indígena & $\sqrt{ }$ & $\sqrt{ }$ & Rural & $\mathrm{X}$ & Indígena & \\
\hline Indígena & $\mathrm{X}$ & $\checkmark$ & Rural & $\checkmark$ & Indígena & \\
\hline Indígena & $\mathrm{X}$ & $\mathrm{X}$ & Ciudad & $\mathrm{X}$ & Indígena & \\
\hline
\end{tabular}

\footnotetext{
${ }^{2} \mathrm{El}$ arraigo a la localidad se puede definir como el apego al lugar geográfico y territorial de origen. Hacer la diferencia entre arraigo a la localidad y lugar de origen resulta importante porque la mayoría de los estudiantes de la UQRoo proviene de comunidades rurales y no de ambientes urbanos, generando migración rural-urbana constante y, en ocasiones, un desapego a la comunidad de origen.

${ }^{3}$ El concepto de ancestralidad denota un proceso relacional con el origen que condensa: el lugar físico y social como territorio de los ancestros; la vida comunitaria, en particular el sistema de cargos y el tequio; y la lengua indígena. A la vez, estas tres dimensiones constituyen ejes de identidad cultural en una dialéctica de adscripción y diferencia (Hernández y Velasco, 2015).

${ }^{4}$ Este indicador muestra el lugar de nacimiento de los estudiantes; aquí se puede mostrar que existen indígenas urbanos, lo que rompe con el imaginario colectivo que perpetúa al indígena en el ambiente rural.
} 


\begin{tabular}{|l|l|l|l|l|l|l|}
\hline Mestizo & $\checkmark$ & $\checkmark$ & Rural & X & Mestiza & $\begin{array}{l}\text { Mestiza } \\
\text { occidental }\end{array}$ \\
\cline { 1 - 5 } Mestizo & X & X & Rural & X & Mestiza & \\
\cline { 1 - 6 } Mestizo & X & X & Ciudad & X & Mestiza & \\
\hline
\end{tabular}

FUENTE: elaboración propia.

Se dieron dos descubrimientos que considero importantes, el primero es que no hubo diferencia entre la autoadscripción y la identidad que sustenta, parece ser que esta coincidencia entre factores es una de las formas más correctas de identificar la pertenencia étnica de las personas. El segundo elemento es que la identidad adoptada no tiene mucho que ver con la afinidad al sistema cultural: no por ser mestizo se desea una cultura occidental y no por ser indígena se desea una cultura originaria. Esta preferencia a un sistema cultural depende de otras características más personales e individuales; sin embargo, es necesario mencionar que el sentimiento de arraigo a la localidad de origen es fundamental para autoidentificarse o para generar sentido de pertenencia a un grupo étnico específico.

El uso de la lengua maya no indicó necesariamente una identidad indígena; por lo tanto, no es posible asegurar entonces que por el lenguaje se tenga pertenencia étnica como se había establecido anteriormente, ya que hay personas que la hablan y se consideran mestizos, así como personas que se consideran indígenas y que no se pueden comunicar en maya.

En la UQRoo se puede concluir que la identidad indígena en los estudiantes es un conjunto interrelacionado de elementos que no pueden ni deben ser separados; aquí radica precisamente una de las dificultades para poder entenderla y analizarla, ya que es una interrelación entre distintos elementos que varían en el contexto urbano o rural.

\section{Del debate identitario en la UIMQRoo}

La UIMQRoo está ubicada en el municipio de José María Morelos, que tiene 56.5\% de la población mayor a 5 años identificado como indígenas, a diferencia del $9.5 \%$ de Othón P. Blanco (INEGI, 2010b), donde se ubica la UQRoo. La cabecera municipal es la zona más urbanizada y existe un predominio de localidades rurales; $72.3 \%$ de la población se encontraba en condición de pobreza y $87.9 \%$ tiene carencia de acceso a la seguridad social, mientras que $29.5 \%$ vio limitado el acceso a la alimentación (Sedesol, 2014).

En 2016, había inscritos un total de 613 alumnos que pertenecen a 8 diferentes licenciaturas, todas con enfoque intercultural y cuya identidad étnica, según un informe interno, estaba distribuida de la siguiente forma: 
Tabla 2. Pertenencia étnica de alumnos inscritos en la UIMQRoo

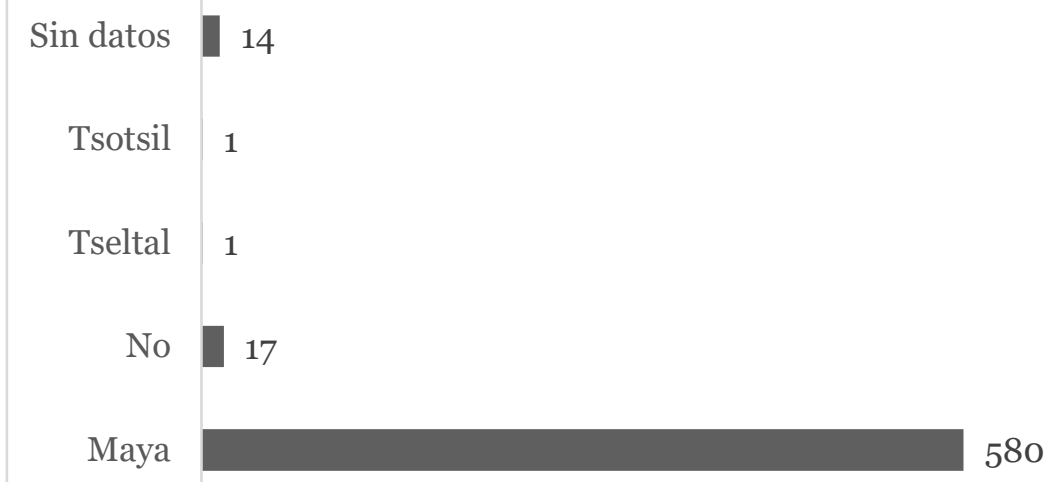

FUENTE: elaboración propia a partir de la base de datos proporcionada por la UIMQRoo.

Esta categorización la hace la propia universidad teniendo de nueva cuenta los indicadores de lengua y autoadscripción, pero hay que poner atención en los 17 estudiantes que indican no tener pertenencia étnica, pues esto no puede ser posible. Probablemente no se identificaban con las opciones del cuestionario y es interesante observar que la opción de ser mestizo tampoco está presente en esta forma de recuperar datos.

En la práctica cotidiana de la UIMQRoo se hace presente un discurso que reflexiona la categoría de indígena y se cuestiona la relación binaria y excluyente de indígena/mestizo,

... que está basado en contrastar criterios de raza, cultura, clase, nación, comunidad, etc., y es para obtener una claridad comparativa en las regiones de México, Mesoamérica y Latinoamérica [...] Es necesario entender que el término identidad puede permanecer constante pero sus valores, significados y usos son variables en el tiempo (Castañeda, 2004: 5-6).

Este tipo de discusión se da en primer lugar en el ambiente académico, pero tiene un impacto en los estudiantes ya que, a lo largo de su estancia escolar, se encarna o se analiza desde su propia experiencia. Se intenta

... comprender la importancia de las múltiples representaciones sobre la concepción de la identidad maya debido a que no somos agentes pasivos, sino actores con agencia. La agencia de los alumnos de la UIMQRoo para aceptar, interpelar y contestar los discursos identitarios, permitiendo de esta manera, tener opciones entre el ser o no ser (Pool, 2012: 8).

$\mathrm{Al}$ hacer una revisión de materiales, la discusión sobre identidad que se desarrolla dentro de la UIMQRoo puede resumirse en tres puntos (Castillo Cocom, 2007; Pool, 2012):

a) La identidad maya es una forma de sentirse y de verse, que tiene que ver con la habilidad de hablar o de entender el idioma y con el nacimiento dentro de localidades ubicadas geográficamente en la zona maya. Ser nativo y usar la lengua es un elemento irrefutable.

b) Se necesita del otro que nombra para existir y es al mismo tiempo un 
invento cultural. De hecho, en algunas narrativas se encuentra que el término maya es creado por los conquistadores, y en ocasiones los pobladores no se consideran como tales, pues es una etiqueta impuesta por el otro. Se nutre de una oposición binaria y antagónica que sólo en la teoría tiene fronteras bien definidas.

c) Respecto al uso, la identidad es un elemento estratégico, que se puede poner o quitar y que es válido en un tiempo y lugar específicos; es decir, se es maya en ciertos lugares y momentos que lo ameritan, y en otros no. Es el actor quien decide cuándo utilizarlo y cuándo no, según sus objetivos, defensas o finalidades.

La UIMRoo permite analizar y debatir las identidades dentro de su contexto local, nacional y mundial, lo que trae como resultado que los estudiantes desarrollen el poder de autodefinirse de forma crítica y de empoderar su voz en su comunidad o sociedad, y no sólo quedarse con la etiqueta que les es impuesta. Se les otorga la posibilidad de cuestionarse y de elegir quiénes quieren ser, en un proceso que no es sencillo.

Esta forma de cuestionar, de deconstruir y reconstruir la identidad tiene resultados que pueden ser positivos o negativos: en cuanto a los positivos puede decirse que son reflejados en los estudiantes que reafirman o se autodenominan mayas; en cambio, los negativos serían aquellos en donde se rehúye o no se revitaliza la cultura maya de forma ya consciente. Un ejemplo es la tesis universitaria de Edwin Pool (2012), en la que se investiga las razones para ser o no ser maya por parte de 16 estudiantes; se encuentran seis variantes:

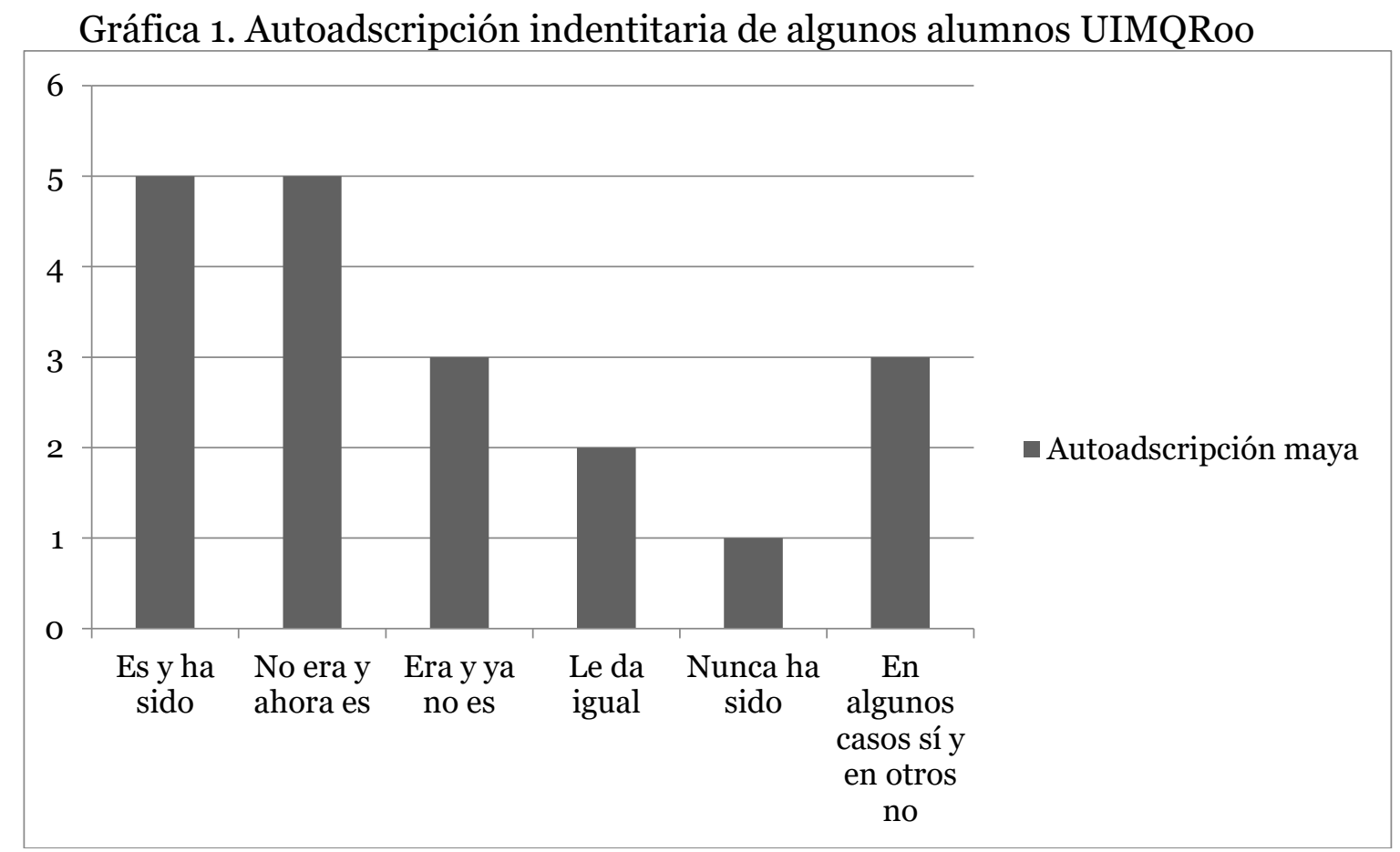

FUENTE: gráfica elaborada a partir de los datos de Pool, 2012: 36-37.

Sin importar el resultado, este ejercicio de reflexividad y de autodeterminación se da gracias a un modelo educativo que pone en tela de juicio la identidad y no la asume como algo natural ni definido, sino precisamente como algo complejo y estratégico. 
Para la identificación se analizan la familia, ancestralidad, imposición, zona geográfica, genotipos y fenotipos, fisiología, etc. Al poner tantas variables y analizarlas, la pertenencia o rechazo se vuelve un proceso consciente y reflexivo por parte de los alumnos, lo que, en caso de que decidan autoadscibirse así, refuerza la identidad, cumpliendo uno de los objetivos de este modelo educativo.

Para corroborar esto, se aplicó la misma metodología y técnicas que en la otra universidad, nuevamente a dos grupos de ocho estudiantes voluntarios pertenecientes a todas las licenciaturas. En el análisis de la información se encuentran menciones a la autoadscripción, ancestralidad y al territorio; y aparece una nueva relación: la identidad impuesta a causa de una hegemonía cultural que es precisamente, el mestizaje.

Por otra parte, para la identidad mestiza no hubo otros datos ni referencias consistentes en los discursos de los estudiantes. No se presentaba una reflexividad tan intensa como en el caso de la indígena; su definición es más bien el no indígena o el discurso de la "mezcla de culturas" que se ha fomentado desde siempre en el sistema educativo; es decir, si no se pertenece a una cultura indígena entonces, por definición, se es mestizo.

Imagen 1. Elementos que configuran la identidad en la UIMQRoo

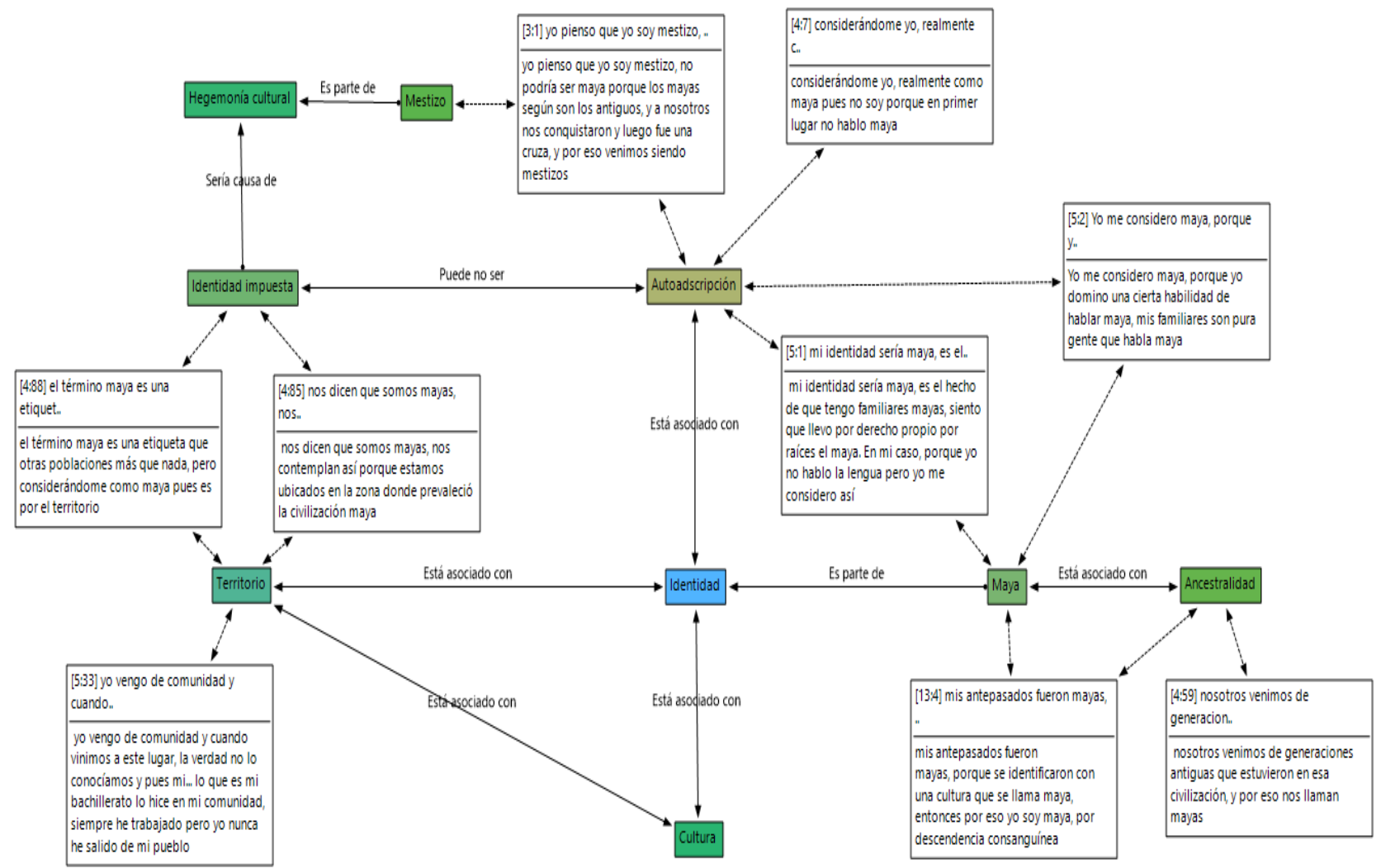

FUENTE: elaboración propia con datos de trabajo de campo. 


\section{Conclusiones}

Para indagar de forma más clara la configuración de la identidad étnica en espacios interculturales, es necesario profundizar y analizar las historias de vida, pues esto pareciera ser un proceso individual que depende de las experiencias e intereses personales de cada sujeto que se ven reflejados en lo social, pero habría que ir descifrando el papel que juegan las relaciones socioculturales en el contexto en que se desenvuelven los estudiantes. Otro descubrimiento es que, si bien la interculturalidad busca fortalecer la relación entre identidad y afinidad cultural, puede generar precisamente lo contrario. Cuando las personas conocen otras culturas e identidades pueden sentirse más atraídos por éstas y paulatinamente abandonar su identidad original, para adoptar otras a lo largo de la vida.

Por último, se debe comprender que la identidad depende de los contextos y de los usos, por lo que se convierte en una característica estratégica y moldeable, sobre todo en el caso de las personas indígenas, a diferencia de las personas mestizas. Los estudiantes se piensan y se asumen como mayas o no según el contexto en el que se deba entrar o salir. Pueden serlo en su comunidad, pero al salir de ella lo evitan; en otras palabras, el ser maya puede ser entendido como una metáfora de una máscara que puede ser utilizada dependiendo de ciertos escenarios y objetivos a lograr.

Es necesario mencionar que sí se generaron cambios a través de este modelo educativo, pero en estudiantes que se consideran pertenecientes a una etnia, en específico a la maya, y que tienen interés en conservar su cultura pues, como se pudo observar, no todos están interesados en esto. Se considera que la educación intercultural que se brinda tiene la abre la posibilidad de ubicar a la cultura maya en espacios urbanos y de difundir sus conocimientos en la ciudad. Sin embargo, queda pendiente la otra parte de cualquier relación intercultural: el interés del mestizo occidental en aprender y dejarse enseñar por quienes supone como inferiores. Sin esta característica será difícil que las prácticas tengan más alcance.

\section{Aceptado el 8 de noviembre de 2017}

\section{Bibliografía}

BARI, María (2002). "La cuestión étnica: aproximaciones a los conceptos de grupo étnico, identidad étnica, etnicidad y relaciones interétnicas”, Cuadernos de Antropología Social. (16), 149-163.

BRAVO, Carlos (1993). "Reelaboración de identidades indígenas en la ciudad de México", Margarita Estrada, Antropología y ciudad. México: CIESAS/UAM.

CASTAÑEDA, Quetzil (2004). "iNo somos indígenas! Una introducción a la identidad maya de Yucatán”, Juan Castillo Comom y Quetzil Castañeda, Estrategias identitarias. Educación y la antropología histórica en Yucatán. Mérida, México: UPN.

CASTILLO COCOM, Juan (2007). "Maya Scenarios: Indian Stories in and out of Contexts", Kroeber Antropological Society. 96, 13-35.

CENEI (2016). Informe 2015. Chetumal: UQRoo. Disponible en: http://cenei.uqroo.mx/

CEPAL (2014). Los pueblos indígenas en América Latina. Infografía publicada el 22 
de septiembre de 2014, disponible en: https://www.cepal.org/es/infografias/los-pueblos-indigenas-en-americalatina.

CHI CANUL, Hilario (2010) “Aquí hablo maya, pero allá debo hablar español. Las luchas de la lengua maya y el español en Naranjal Poniente. Desafíos para la enseñanza de lengua maya en la educación superior”. Revista Tellus, 10 (19), $211-224$.

DURÁN, Leonel (2005). "El etnodesarrollo y la promoción cultural en México", Conaculta (ed.), Antología sobre culturas populares e indígenas. Vol. II, México: Conaculta, pp. 97-108.

EPSTEIN, Gil y Odelia Heizler (2015). "Ethnic Identity: A Theoretical Framework”, IZA Journal of Migration. 4 (9).

ESPÍN, Julia et al. (1998). "Elaboración de un cuestionario para medir la identidad étnica y la aculturación en la adolescencia”, Revista de Educación. (315), 227249.

GIMÉNEZ, Gilberto (2007). Estudios sobre la cultura y las identidades sociales. México: UI/ITESO/UV/UdeG.

GUTIÉRREZ CHONG, Natividad y Luz Valdés (2015). Ser indígena en México. Raíces y derechos. Encuesta Nacional de Indígenas. México: UNAM.

HALL, Stuart (2014). Sin garantías. Colombia: Editorial Universidad del Cauca.

HERNÁNDEZ, Héctor y Ana María Chávez (2007). "La definición de la población indígena en el censo de población del año 2000 en México", Héctor Hernández (coord.), Los indios de México en el siglo XXI. Cuernavaca: UNAM/Centro Regional de Estudios Multidisciplinares, disponible en: http://132.248.9.34/libroe_2007/1118539/05_02.pdf.

HERNÁNDEZ, María Eugenia y Laura Velasco (2015). "La etnicidad cuestionada: ancestralidad en las hijas e hijos de inmigrantes indígenas oaxaqueños en Estados Unidos", Migraciones Internacionales. 8 (2), 134-163.

INEGI (2010a). Principales resultados del censo de población y vivienda de 2010. México, disponible

en: http://www.inegi.gob.mx/prod serv/contenidos/espanol/bvinegi/producto s/censos/poblacion/2010/princi_result/cpv2010_principales_resultadosI.p df.

----- (210b). Principales resultados del censo de población y vivienda de 2010. Quintana Roo. México, disponible en: http://www.inegi.org.mx/prod_serv/contenidos/espanol/bvinegi/producto s/censos/poblacion/2010/princi result/qroo/23 principales resultados c pv2010.pdf.

----- (2015). Encuesta intercensal 2015. Principales resultados. México, disponible http://www.beta.inegi.org.mx/contenidos/proyectos/enchogares/especiales /intercensal/2015/doc/eic2015 resultados.pdf.

------ (s/f). La población indígena en México. México, disponible en: http://www.inegi.org.mx/inegi/contenidos/espanol/eventos/vigenero/dia2 9/panel4 mesas/Poblacion indigena/P indigena-en-Mexico.pdf.

MATEOS, Laura y Gunther Dietz (2016). "Universidades interculturales en México. Balance crítico de la primera década”, Revista Mexicana de Investigación 
Educativa. 21 (70), 683-690.

MENDOZA, Rosa (2017). "Inclusión educativa por interculturalidad: implicaciones para la educación de la niñez indígena”, Perfiles Educativos. 39 (158), 52-69.

OLIVERA RODRÍGUEZ, Inés (2014). “¿Desarrollo o bien vivir? Repensando la función social de la Universidad Intercultural desde el cuestionamiento al efecto educativo", Anthropologica. 32 (33), 179-207, disponible en: http://www.redalyc.org/articulo.oa?id=88636924009.

POOL, Edwin (2012). Discursos identitarios: el sakbej de la mayanidad. Tesis para obtener el grado de licenciado en Lengua y Cultura, UIMQRoo, Quintana Roo.

REBOLLEDO, Nicolás (2005). "Interculturalismo y autonomía. Las universidades indígenas y las políticas de alteridad", César Navarro (coord.), La mala educación en tiempos de la derecha. Política y proyectos del gobierno de Vicente Fox. México: Porrúa/UPN, 328 p.

SEDESOL (2014). Informe anual sobre la situación de pobreza y rezago social. Quintana Roo. Pobreza municipal. México: Sedesol/Coneval, disponible en: http://www.sedesol.gob.mx/work/models/SEDESOL/Informes pobreza/2 014/Municipios/Quintana Roo/Quintana Roo 006.pdf el día 7 de marzo de 2016.

UNAM (2008). Los pueblos indígenas y los indicadores de bienestar y desarrollo. Pacto del Pedregal. Distrito Federal. 